\title{
Genetic profiling of 2,683 Vietnamese genomes from non-invasive prenatal testing data
}

Ngoc Hieu Tran ( $\nabla$ hieutran1985@gmail.com )

University of Waterloo https://orcid.org/0000-0001-5186-8852

Thanh Binh Vo

Gene Solutions

Van Thong Nguyen

Hung Vuong Hospital

Nhat Thang Tran

University Medical Center, Ho Chi Minh city

Thu-Huong Nhat Trinh

Tu Du Hospital

Hong-Anh Thi Pham

Gene Solutions

Hong Thuy Dao

Gene Solutions

Ngoc Mai Nguyen

Gene Solutions

Yen-Linh Thi Van

Gene Solutions

Vu Uyen Tran

Gene Solutions

Hoang Giang Vu

Gene Solutions

Quynh-Tram Nguyen Bui

Gene Solutions

Phuong-Anh Ngoc Vo

Gene Solutions

Huu Nguyen Nguyen

Gene Solutions

Quynh-Tho Thi Nguyen

Medical Genetics Institute

Thanh-Thuy Thi Do

Medical Genetics Institute

Phuong Cao Thi Ngoc 
Lund University

\section{Dinh Kiet Truong}

Medical Genetics Institute

\section{Hoai-Nghia Nguyen}

University of Medicine and Pharmacy at Ho Chi Minh city

\section{Hoa Giang}

Medical Genetics Institute

\section{Minh Duy Phan}

The University of Queensland

\section{Research article}

Keywords: genome sequencing, population genetics, non-invasive prenatal testing

Posted Date: January 3rd, 2020

DOl: https://doi.org/10.21203/rs.2.20017/v1

License: (c) (i) This work is licensed under a Creative Commons Attribution 4.0 International License. Read Full License 


\section{Genetic profiling of 2,683 Vietnamese genomes from non-invasive}

\section{2 prenatal testing data}

3 Ngoc Hieu Tran ${ }^{1,2, *}$, Thanh Binh Vo ${ }^{1,3}$, Van Thong Nguyen ${ }^{4}$, Nhat Thang Tran ${ }^{5}$, Thu-Huong Nhat

4 Trinh ${ }^{6}$, Hong-Anh Thi Pham ${ }^{1,3}$, Hong Thuy Dao ${ }^{1,3}$, Ngoc Mai Nguyen ${ }^{1,3}$, Yen-Linh Thi Van ${ }^{1,3}$, Vu

5 Uyen Tran ${ }^{1,3}$, Hoang Giang Vu ${ }^{1,3}$, Quynh-Tram Nguyen Bui ${ }^{1,3}$, Phuong-Anh Ngoc Vo ${ }^{1,3}$, Huu

6 Nguyen Nguyen ${ }^{1,3}$, Quynh-Tho Thi Nguyen ${ }^{3}$, Thanh-Thuy Thi Do ${ }^{3,7}$, Phuong Cao Thi Ngoc ${ }^{3,7}$,

7 Dinh Kiet Truong ${ }^{3}$, Hoai-Nghia Nguyen ${ }^{8, *}$, Hoa Giang ${ }^{1,3, \star}$, Minh-Duy Phan ${ }^{1,3,9, *}$

$9 \quad{ }^{1}$ Gene Solutions, Vietnam

$10{ }^{2}$ David R. Cheriton School of Computer Science, University of Waterloo, Canada

11 3Medical Genetics Institute, Vietnam

$12 \quad{ }^{4}$ Hung Vuong hospital, Vietnam

13 Enniversity Medical Center, Ho Chi Minh city, Vietnam

$14{ }^{6} \mathrm{Tu}$ Du hospital, Vietnam

$15{ }^{7}$ Division of Molecular Hematology, Lund Stem Cell Center, Lund University, Lund, Sweden

16 'University of Medicine and Pharmacy at Ho Chi Minh city, Vietnam

$17{ }^{9}$ School of Chemistry and Molecular Biosciences, The University of Queensland, Brisbane,

18 Australia

19

20

${ }^{*}$ Corresponding authors.

21 Emails: nh2tran@uwaterloo.ca (Ngoc Hieu Tran); m.phan1@uq.edu.au (Minh-Duy Phan);

22 gianghoa@gmail.com (Hoa Giang); nhnghia81@gmail.com (Hoai-Nghia Nguyen). 


\section{Abstract}

25 Background: The under-representation of Vietnamese ethnic groups in existing genetic 26 databases and studies have undermined our understanding of the genetic variations and

27 associated traits or diseases in the population. Cost and technology limitations remain the challenges in performing large-scale genome sequencing projects in Vietnam and many developing countries. Non-invasive prenatal testing (NIPT) data offers an alternative untapped resource to study genetic variations in the Vietnamese population.

31 Results: We analyzed the low-coverage genomes of 2,683 pregnant Vietnamese women using

32 their NIPT data and identified a comprehensive set of $8,054,515$ single-nucleotide

33 polymorphisms, among which $8.2 \%$ were new to the Vietnamese population. Our study also

34 revealed 24,487 disease-associated genetic variants and their allele frequency distribution, 35 especially five pathogenic variants for prevalent genetic disorders in Vietnam. We also observed 36 major discrepancies in the allele frequency distribution of disease-associated genetic variants

37 between the Vietnamese and other populations, thus highlighting a need for genome-wide 38 association studies dedicated to the Vietnamese population.

39 Conclusions: We have demonstrated a successful analysis of NIPT data to reconstruct the

40 Vietnamese genetic profiles. This application provides a powerful yet cost-effective approach for 41 large-scale population genetic studies.

42 Keywords: genome sequencing; population genetics; non-invasive prenatal testing 


\section{Background}

45 Following the successful initiative of the 1000 genomes project [1], several large-scale genome and exome sequencing projects have been conducted, either as international collaboration

47 efforts such as EXACT [2], gnomAD [3], or for a specific country or population [4-8]. Those projects have provided comprehensive profiles of human genetic variation in some populations, paving the way for unprecedented advance in treatment of common genetic diseases. However, the lack of diversity and the under-representation of several populations in genome sequencing

51 projects and genome-wide association studies (GWAS) have increasingly become a critical

52 problem [9,10]. For instance, Gurdasani et al. found that the representation of ethnic groups in

53 GWAS was significantly biased, with nearly $78 \%$ of the participants having European ancestries, 54 whereas the two major populations, Asian and African, only accounted for $11 \%$ and $2.4 \%$, 55 respectively [10]. Vietnam has a population of 96.5 million, the $15^{\text {th }}$ highest in the world and the $569^{\text {th }}$ highest in Asia. Yet there was merely one dataset of 99 Vietnamese individuals that had 57 been studied as part of the 1000 genomes project (population code KHV, the Kinh ethnic group

58 in Ho Chi Minh City, Vietnam). A recent study has sequenced genomes and exomes of another 59305 individuals to further expand the Vietnamese genetic database [11]. However, costs and technologies to perform large-scale genome sequencing projects still remain a challenge for most developing countries, including Vietnam.

An alternative approach has been proposed recently to re-use the low-coverage genome sequencing data from non-invasive prenatal testing (NIPT) for large-scale population genetics studies [12,13]. NIPT is a method that sequences cell-free DNA from maternal plasma at an ultra-low depth of 0.1-0.2x to detect fetal aneuploidy [14]. By combining a sufficiently large number of NIPT samples, one could obtain a good representation of the population genetic

67 variation. The benefits of re-using NIPT data for population genetics are manifold. First, the data can be re-used at no extra cost given the approval and consent of the participants. As one of 
the most rapidly adopted genetic tests, NIPT has been successfully established and become a standard screening procedure with thousands to millions of tests performed world-wide, including many developing countries such as Vietnam [14]. Using NIPT data for population genetic studies may also reduce privacy concerns since the genetic variants can only be analyzed by aggregating a large number of samples and the results can only be interpreted at the population level. A single sample tells little about the genetic information of an individual due to low sequencing depth. Last but not least, previous studies have suggested that sequencing a large number of individuals at a low depth might provide more accurate inferences of the population genetic structure than the traditional approach of sequencing a limited number of individuals at a higher depth, especially when the budget is limited $[15,16]$.

In this paper, we presented the first study of Vietnamese genetic variations from non-invasive prenatal testing data, and to the best of our knowledge, the third of such kinds in the world $[12,13]$. We analyzed the NIPT data of 2,683 pregnant Vietnamese women to identify genetic variants and their allele frequency distribution in the Vietnamese population. We also studied the relationships between the Vietnamese genetic profile and common genetic disorders, and discovered pathogenic variants related to prevalent diseases in Vietnam. Finally, we highlighted the differences in the distribution of disease-associated genetic variants between the Vietnamese and other populations, thus highlighting a need for genome-wide association studies dedicated to the Vietnamese population.

\section{Results}

\section{Data collection}

A total of 2,683 pregnant Vietnamese women who performed non-invasive prenatal testing during the period from 2018-2019 at the Medical Genetics Institute, Vietnam, were recruited to the study. The participants have approved and given written consent to the anonymous re-use of their genomic data for the study. All information of the participants is confidential and not 
available to the authors, except the records that their NIPT and pregnancy results are normal.

The study was approved by the institutional ethics committee of the University of Medicine and Pharmacy, Ho Chi Minh city, Vietnam. The whole genome of each participant was sequenced to an average of 3.6 million paired-end reads of $2 \times 75 \mathrm{bp}$, which corresponds to a sequencing depth of $0.17 x$ per sample.

\section{Genome coverage and sequencing depth of the NIPT dataset}

Data pre-processing was first performed on each of 2,683 samples and the results were stored in binary alignment map (BAM) format, one BAM file per sample. The data pre-processing steps include: quality control of raw data using FastQC [17], trimmomatic [18]; alignment of paired-end reads to the human reference genome (build GRCh38) using bwa [19], samtools [20], MarkDuplicates [21]; and summary of alignment results using Qualimap [22], bedtools [23], IGV [24]. The quality of raw data and alignment results are presented in Supplementary Figures S1 and S2. Raw data showed high sequencing quality, no error or bias was observed. The mapping quality and insert size distributions followed closely what expected across the reference genome. The overall sequencing error rate was about $0.3 \%$. More details of the data pre-processing steps can be found in the Methods section.

The average genome coverage and depth were $14.59 \%$ and $0.17 x$ per sample, respectively, and aggregated to $95.09 \%$ and $462 x$ across 2,683 samples (Figure 1a). Although the sequencing depth per sample was low, there might be more than one read from the same sample overlapping at a genome position. This problem may affect the estimation of allele frequency because the estimation is based on the assumption that a sample may contribute only 0 or 1 allele (read) at any given genome position [12,13]. For instance, we found that the average percentage of genome positions with depth $2 x$ (i.e. covered by two overlapping reads) in a sample was $1.75 \%$ (Figure 1a). These overlapping reads occurred randomly across the reference genome and the samples. At any genome position, there were on average 47 out of 
2,683 samples that each contributed two reads (Supplementary Figure S3). To address this

120 problem, we followed a filtering strategy from previous studies $[12,13]$ to keep only one read if

121 there were overlapping reads in a sample. Thus, for every genome position, each sample could

122 only contribute up to one read, and when the samples were aggregated, all reads at any

123 position were obtained from different samples. In addition, we also removed alignments with low

124 mapping quality scores (MAPQ $<30)$.

125 After the filtering step, the sequencing depth was reduced from $0.17 x$ to $0.12 x$ per sample. The aggregated sequencing depth of 2,683 samples was 364x and the genome coverage was

$12791.56 \%$ (Figure 1a). The distributions of genome coverage and sequencing depth are presented

128 in Figures $1 \mathrm{~b}-\mathrm{d}$. The sequencing depth was approximately uniform across the reference

129 genome, except for low-mappability regions and chromosome $\mathrm{Y}$. The distributions of sequencing depth and MAPQ score also closely followed the mappability of the human reference genome obtained from Umap [25]. The average sequencing depth of chromosome $Y$

132 was about $2.1 \%$ that of the whole genome, consistent with the proportion of fetal DNA in NIPT 133 samples (8-10\%) [14].

\section{Variant calling and validation}

135 We aggregated 2,683 samples into one and used Mutect2 from GATK [26,27] for variant calling 136 and allele frequency estimation. In addition to its main function of somatic calling, Mutect2 can 137 also be used on data that represents a pool of individuals, such as our NIPT dataset, to call 138 multiple variants at a genome site $[12,13,28]$. The called variants were further checked against 139 strand bias, weak evidence, or contamination using FilterMutectCalls. The allele frequencies 140 were estimated based on the numbers of reads aligned to the reference and the alternate 141 alleles. For validation, we compared our NIPT call set to the KHV (Kinh in Ho Chi Minh City, 142 Vietnam) and EAS (East Asian) populations from the 1000 genomes project [1], as well as the 143 dbSNP database (version 151, [29]). 
144 We identified a total of $8,054,515$ SNPs from the NIPT dataset. The transition to transversion

145 ratio was 2.0 over the whole genome and 2.8 over protein coding regions, which was similar to 146 the observed ratios from previous genome or exome sequencing projects. As expected, a 147 majority of these SNPs, $7,390,020$ or $91.8 \%$, had been reported earlier in the KHV call set 148 (Figure 2a). Since the KHV population only had 99 individuals, we further looked into its 149 common SNPs that were shared by at least two individuals. We found that the NIPT call set 150 recovered $90.5 \%$ of the KHV common SNPs (Supplementary Figure S4; 6,889,016 / 7,609,526 $151=90.5 \%)$. This sensitivity is in line with the genome coverage reported earlier in Figure 1a. An 152 important advantage of NIPT data is the ability of sampling a large number of individuals to 153 better represent a population and to accurately estimate the allele frequency. We found a strong 154 Pearson correlation of $98.8 \%$ between the allele frequency of the NIPT call set and that of the $155 \mathrm{KHV}$ call set (Figure 2b). Furthermore, thanks to its larger sample size, the NIPT allele 156 frequency indeed showed better resolution than the KHV one, as evidenced by vertical trails in 157 Figure $2 \mathrm{~b}$ or a zoomed-in view in Supplementary Figure S5.

Our NIPT call set included 664,495 (8.2\%) SNPs that had not been reported in the KHV call set. 159 Among them, $67,153(0.8 \%)$ were found in the EAS call set, another $517,020(6.4 \%)$ were found 160 in the dbSNP database, and the remaining 80,322 (1.0\%) were novel SNPs (Figure 2a).

161 Majority of those SNPs had allele frequencies less than 10\%. The overall allele frequency 162 distribution of our NIPT call set is presented in Figure 2c.

163 We used VEP (Variant Effect Predictor [30]) to analyze the effects of 8,054,515 variants in the 164 NIPT call set (Figure $2 \mathrm{~d}$ ). About $1.5 \%$ of the SNPs were located in the coding and UTR regions, $16512 \%$ in the upstream and downstream regions, and $4 \%$ in the TF regulatory regions. More than $16680 \%$ of the SNPs were located in the intron and intergenic regions. We also noted that the new 167 SNPs and those in KHV had similar proportions of coding, UTR, upstream and downstream, 168 and TF regulatory regions (Figure 2d). 
170 We searched the NIPT call set against the ClinVar database (version 20191105, [31]) to explore

171 the associations between Vietnamese genomic variants and common genetic diseases. We

172 identified 24,487 SNPs with ClinVar annotations that have been reviewed by at least one

173 research group (Table 1). Among them, five SNPs were classified as "Pathogenic" or "Likely

174 pathogenic", 117 SNPs were found to affect a "drug response", and a majority of the remaining

175 were classified as "Benign" or "Likely benign". We also noted that 391 ClinVar-annotated SNPs

176 (1.6\%), including 1 pathogenic SNP, had not been reported in the KHV call set.

177 Table 2 and Supplementary Table S1 present the details of five pathogenic SNPs identified in

178 our NIPT call set. Their associated genetic diseases include: erythropoietic protoporphyria, non-

179 syndromic genetic deafness, Joubert syndrome, hemochromatosis type 1, and 5-alpha

180 reductase deficiency. The SNP rs9332964 C>T in SRD5A2, which is associated with 5-alpha

181 reductase deficiency, had not been reported in the KHV call set. 5-alpha reductase deficiency is

182 an autosomal recessive disorder that affects male sexual development. This SNP is rare in the

183 world and East Asia populations, but was found to be more common in the Vietnamese

184 population (allele frequency of $0.05 \%, 0.67 \%$, and $2.90 \%$, respectively). This SNP had also

185 been reported in a recent study [11] at a very low allele frequency of $1.36 \%$.

186 We noticed that the allele frequencies of the five pathogenic SNPs varied considerably between

187 the Vietnamese, the East Asia, and the world populations (Table 2). For instance, the SNP

188 rs72474224 C>T in GJB2 is commonly liked to non-syndromic hearing loss and deafness, which

189 is also the most prevalent genetic disorder in the Vietnamese population. We found that its

190 allele frequency in the Vietnamese population was $60 \%$ higher than in the East Asia population,

191 which in turn was an order of magnitude higher than in the world population $(13.40 \%, 8.35 \%$,

192 and $0.76 \%$, respectively). The allele frequency was consistent with the estimated carrier

193 frequency of 1 in 5 in the Vietnamese population. Similarly, the allele frequency of rs2272783 
$\mathrm{A}>\mathrm{G}$ in $\mathrm{FECH}$, which is associated with erythropoietic protoporphyria, was nearly three times higher in the Vietnamese and East Asia populations than in the world population $(28.10 \%$, $32.57 \%$, and $11.23 \%$, respectively). The prevalence of this pair of SNP and disease in East and

197 Southeast Asia has been reported previously in [32]. On the other hand, the allele frequency of rs1799945 C>G in HFE, which is associated with hemochromatosis type 1, was about two and three times lower in the Vietnamese and East Asia populations than in the world population (5.10\%, 3.41\%, and 10.82\%, respectively). Such discrepancies were also observed for "Benign" variants, e.g., those related to autosomal recessive non-syndromic hearing loss (Supplementary

202 Table S2). The variations strongly suggest that population-specific genome-wide association studies are required to provide a more accurate understanding of the clinical significance of genetic variants and the true disease prevalence in the Vietnamese population.

\section{Discussion}

In this study, we analyzed the genomes of 2,683 pregnant Vietnamese women from their noninvasive prenatal testing data. The genomes were originally sequenced at a low depth of approximately $0.17 x$ per sample for the purpose of fetal aneuploidy testing [14]. Here we combined the 2,683 samples to a total sequencing depth of $364 x$ and performed variant calling and analysis for the Vietnamese population. We identified a comprehensive set of $8,054,515$ SNPs at a high level of sensitivity and accuracy: $90.5 \%$ of Vietnamese common SNPs were recovered; 99\% of identified SNPs were confirmed in existing databases; and a strong correlation of $98.8 \%$ to known allele frequencies. The results were exciting given that the total

214 sequencing depth of our dataset, 364x, was merely equivalent to sequencing 20 individuals at a 215 moderate depth of 20x. It also suggests that there is still plenty of room for improvement by 216 increasing the number of NIPT samples. For instance, Liu et al. have demonstrated a large217 scale population genetic analysis based on hundreds of thousands of NIPT samples for the 218 Chinese population [12]. 
Another benefit of using NIPT data is cost-effective. In our study, the dataset was re-used at no cost with written consent from the participants. The whole analysis pipeline was done within a week on a cloud computing platform for a few hundred dollars. Thus, the overall cost was

222 negligible compared to that of a typical genome sequencing project. The cost advantage of this 223 approach may play a major role in large-scale genome sequencing projects, especially in 224 developing countries where technologies and resources are still limited.

225 Our study revealed 24,487 disease-associated genetic variants, especially five pathogenic variants for prevalent genetic disorders in the Vietnamese population. We also found major 227 discrepancies in the allele frequency distribution of genetic variants between the Vietnamese, 228 the East Asia, and the world populations. Thus, a comprehensive genetic profile and genome229 wide association studies dedicated to the Vietnamese population are highly desired. Knowing 230 the distribution of genetic disorders in the population will be useful for public health policy and 231 planning, preventive medicine, early genetic screening strategies, etc.

232 Some technical and design limitations in our study could be addressed in future research to 233 improve the application of NIPT data in population genetics studies. First, currently there is no 234 variant calling tool that is designed specifically for NIPT data. Here we used Mutect2 and 235 previous studies also used similar somatic calling tools with the purpose of identifying all 236 possible variants at a genome site $[12,13]$. Thus, we took a conservative approach to consider 237 only SNPs but not indels to ensure a reliable call set. Another limitation was the exclusion of 238 chromosome $\mathrm{Y}$ due to its low coverage as a result of limited amount of fetal DNA in NIPT 239 samples. This problem could be addressed by increasing the number of samples to obtain 240 enough sequencing coverage for reliable variant calling. NIPT data is also biased by sex, with 241 only $~ 5 \%$ of the data coming from male population (assuming a 10\% cell-free fetal DNA fraction 242 with $50 \%$ male fetuses). In general, sufficiently large sample size is essential to use NIPT data 
243 for population genetics research. Thus, privacy policy, code of ethics, and standards of practice

244 need to be established to protect the confidential information and data of the participants.

\section{Conclusions}

246 We showed that non-invasive prenatal testing data could be reliably used to reconstruct the

247 genetic profile of the Vietnamese population. Our study identified pathogenic variants for

248 prevalent genetic diseases in the Vietnamese population and called for a need for population-

249 specific genome-wide association studies. The results demonstrated that non-invasive prenatal

250 testing data provides a valuable and cost-effective resource for large-scale population genetic

251 studies.

\section{Methods}

\section{Sample preparation}

254 Cell-free DNA (cfDNA) in maternal plasma was extracted using MagMAX Cell-Free DNA 255 Isolation Kit from Thermo Fisher Scientific (Waltham, MA, USA). Library preparation was done 256 using NEBNext Ultra II DNA Library Prep Kit from New England BioLabs (Ipswich, MA, USA).

257 The samples were sequenced on the NextSeq 550 platform using paired-end $2 \times 75$ bp Reagent 258 Kit from Illumina (San Diego, CA, USA).

\section{Bioinformatics analysis pipeline}

260 Quality check of raw sequencing data was performed using FastQC (version 0.11.8, [17]).

261 Paired-end reads were trimmed to $75 \mathrm{bp}$, adapters ("TruSeq3-PE-2.fa”) and low-quality bases

262 were removed using trimmomatic (version $0.39,[18]$ ). We only kept pairs with both reads

263 surviving the trimming (about $95.7 \%$ of the dataset). The reads were then aligned to the human

264 reference genome, build GRCh38 (hg38), using bwa mem (version 0.7.17-r1188, [19]).

265 Supplementary hits were marked as secondary for Picard compatibility. Alignment results were 
sorted and indexed using samtools (version 1.9, [20]). Potential PCR duplicates were marked

267 using MarkDuplicates from GATK (version 4.1.1.0, [26]).

268 Alignments with mapping quality scores less than 30 were discarded. In-house Python scripts 269 were developed to mark overlapping alignments and to keep only one of them. Qualimap

270 (version 2.2.1, [22]), bedtools (version 2.25.0 [23]), and IGV (version 2.4.19, [24]) were used to

271 summarize the alignment results and to calculate genome coverage and sequencing depth.

272 Mutect2 from GATK (version 4.1.1.0 [26,27]) was used in tumor-only mode for variant calling. All

273 samples were assigned the same sample name to combine them before variant calling.

274 FilterMutectCalls was used to exclude variants with weak evidence, strand bias, or

275 contamination. bcftools (version 1.9, [33]) was used to filter, summarize, and compare VCF

276 (Variant Call Format) files. VEP (version 98, [30]) was used to predict the effects of variants and

277 to annotate them against dbSNP (version 151, [29]) and ClinVar (version 20191105, [31])

278 databases.

279 The whole analysis pipeline and parameter settings can be found in the attached Python scripts.

280 Declarations

281 Ethics approval and consent to participate

282 The study was approved by the institutional ethics committee of the University of Medicine and 283 Pharmacy, Ho Chi Minh city, Vietnam. The participants who performed NIPT triSure at Medical

284 Genetics Institute, Vietnam, have approved and given written consent to the anonymous re-use 285 of their genomic data for this study.

286 Consent for publication

287 All authors have read and approved the manuscript for publication.

\section{Availability of data and materials}


289 The NIPT variant call set and Python scripts for the bioinformatics analysis pipeline can be 290 found on GitHub: https://github.com/nh2tran/NIPT_WGS

\section{Competing interests}

292 NHT, TBV, HATP, HTD, NMN, YLTV, VUT, HGV, QTNB, PANV, HNN, HG and MDP are current

293 employees of Gene Solutions, Vietnam. The other authors declare no competing interests.

\section{Funding}

295 This study was funded by Gene Solutions, Vietnam. The funder did not have any additional role

296 in the study design, data collection and analysis, decision to publish, or preparation of the

297 manuscript.

\section{Authors' contributions}

299 TBV, HATP, HTD, NMN, YLTV, VUT, HGV, QTNB, PANV performed experiments.

300 VTN, NTTM, THNT, TTTD recruited patients and performed clinical analysis.

301 HNN, QTTN, PCTN, DKT designed experiments and analyzed data.

302 HNN supervised the project.

$303 \mathrm{NHT}, \mathrm{HG}, \mathrm{MDP}$ analyzed the data and wrote the manuscript, designed experiments and 304 analyzed sequencing data.

305 Acknowledgements

306 The authors thank Dr. Kwok Pui Choi for critical reading of the manuscript. NHT was partially

307 supported by the Canada NSERC grant (OGP0046506) and the Canada Research Chair

308 program. 
309

310

311

312

313

314

\section{References}

1. The 1000 Genomes Project Consortium. A global reference for human genetic variation. Nature 2015;526:68-74.

2. Lek $\mathrm{M}$ et al. Analysis of protein-coding genetic variation in 60,706 humans. Nature 2016;536:285-91.

3. Karczewski KJ et al. Variation across 141,456 human exomes and genomes reveals the spectrum of loss-of-function intolerance across human protein-coding genes. bioRxiv 2019; doi: $10.1101 / 531210$.

4. The UK10K Consortium. The UK10K project identifies rare variants in health and disease. Nature 2015;526:82-90.

5. Gudbjartsson DF et al. Large-scale whole-genome sequencing of the Icelandic population. Nat Genet. 2015;47:435-44.

6. Maretty $L$ et al. Sequencing and de novo assembly of 150 genomes from Denmark as a population reference. Nature 2017;548:87-91.

7. The Genome of the Netherlands Consortium. Whole-genome sequence variation, population structure and demographic history of the Dutch population. Nat Genet. 2014;46:814-25.

8. Wu D et al. Large-Scale Whole-Genome Sequencing of Three Diverse Asian Populations in Singapore. Cell 2019; 179:736-749.

9. Editorial. Diversity matters. Nat Rev. Genet. 2019;20:495.

10. Gurdasani D, Barroso I, Zeggini E, Sandhu MS. Genomics of disease risk in globally diverse populations. Nat Rev Genet. 2019;20:520-535.

11. Le VS et al. A Vietnamese human genetic variation database. Hum Mutat. 2019;40:16641675. 
12. Liu S et al. Genomic Analyses from Non-invasive Prenatal Testing Reveal Genetic Associations, Patterns of Viral Infections, and Chinese Population History. Cell 2018;175:347-359.

13. Budis $\mathrm{J}$ et al. Non-invasive prenatal testing as a valuable source of population specific allelic frequencies. J Biotechnol. 2019;299:72-78.

14. Phan MD et al. Establishing and validating noninvasive prenatal testing procedure for fetal aneuploidies in Vietnam. J Matern Fetal Neonatal Med. 2019;32:4009-4015.

15. Li Y, Sidore C, Kang HM, Boehnke M, Abecasis GR. Low-coverage sequencing: implications for design of complex trait association studies. Genome Res. 2011;21:940-51.

16. Fumagalli M. Assessing the effect of sequencing depth and sample size in population genetics inferences. PLoS One 2013;8:e79667.

\section{7. https://www.bioinformatics.babraham.ac.uk/projects/fastac/}

18. Bolger AM, Lohse M, Usadel B. Trimmomatic: A flexible trimmer for Illumina sequence data. Bioinformatics. 2014;30:2114-20.

19. Li H. Aligning sequence reads, clone sequences and assembly contigs with BWA-MEM. arXiv:1303.3997v2 [q-bio.GN].

20. Li H et al. The Sequence Alignment/Map format and SAMtools. Bioinformatics. 2009;25:2078-9.

21. http://broadinstitute.github.io/picard/

22. Okonechnikov K, Conesa A, Garcia-Alcalde F. Qualimap 2: advanced multi-sample quality control for high-throughput sequencing data. Bioinformatics. 2016;32:292-4.

23. Quinlan AR, Hall IM. BEDTools: a flexible suite of utilities for comparing genomic features. Bioinformatics. 2010;26:841-2.

24. Robinson JT et al. Integrative Genomics Viewer. Nat Biotechnol. 2011;29:24-6.

25. Karimzadeh M, Ernst C, Kundaje A, Hoffman MM. Umap and Bismap: quantifying genome and methylome mappability. Nucleic Acids Res. 2018;46:e120. 
26. Van der Auwera GA et al. From FastQ data to high confidence variant calls: the Genome Analysis Toolkit best practices pipeline. Curr Protoc Bioinformatics. 2013;43:11.10.1-33.

27. Cibulskis $\mathrm{K}$ et al. Sensitive detection of somatic point mutations in impure and

374 Figure Legends

375 Figure 1. Distributions of genome coverage and sequencing depth of the NIPT dataset. (a) 376 heterogeneous cancer samples. Nat Biotechnol. 2013;31:213-9.

28. https://software.broadinstitute.org/gatk/documentation/article?id=11136\#2.1

29. Sherry ST et al. dbSNP: the NCBI database of genetic variation. Nucleic Acids Res. $2001 ; 29: 308-11$.

30. McLaren W. The Ensembl Variant Effect Predictor. Genome Biol. 2016;17:122.

31. Landrum MJ et al. ClinVar: public archive of relationships among sequence variation and human phenotype. Nucleic Acids Res. 2014;42:D980-5.

32. Gouya $L$ et al. Contribution of a common single-nucleotide polymorphism to the genetic predisposition for erythropoietic protoporphyria. Am J Hum Genet. 2006;78:2-14.

33. Li H. A statistical framework for SNP calling, mutation discovery, association mapping and population genetical parameter estimation from sequencing data. Bioinformatics. $2011 ; 27: 2987-2993$. Average genome coverage and sequencing depth per sample and from all samples combined.
(b) Summary histogram of sequencing depth over all genome positions. (c) Distribution of sequencing depth per chromosome. (d) IGV tracks of sequencing depth, bwa MAPQ score, and Umap k50 mappability across the whole genome.

Figure 2. Summary of the NIPT call set. (a) Venn diagram comparison between the NIPT call set, the KHV and EAS call sets from the 1000 genomes project, and the dbSNP database. The percentages were calculated with respect to the NIPT call set. (b) Scatter plot comparison of 
383 allele frequency estimated from the NIPT and the KHV call sets. (c) Allele frequency distribution 384 of the NIPT call set. (d) Distribution of locations and effects of variants in the NIPT call set. 
(a)

\begin{tabular}{lrr}
\hline & Raw data & After filter \\
\hline Coverage and depth per bam & & \\
Genome coverage & $14.59 \%$ & $12.17 \%$ \\
Positions with depth 1x & $12.60 \%$ & $12.17 \%$ \\
Positions with depth 2x & $1.75 \%$ & $0.00 \%$ \\
Positions with depth 3x & $0.20 \%$ & $0.00 \%$ \\
Positions with depth >= 4x & $0.05 \%$ & $0.00 \%$ \\
Average depth & 0.17 & 0.12 \\
\hline Aggregating 2,683 sam ples & & \\
Genome coverage & $95.09 \%$ & $91.56 \%$ \\
Average depth & 462 & 364 \\
\hline
\end{tabular}

(b)

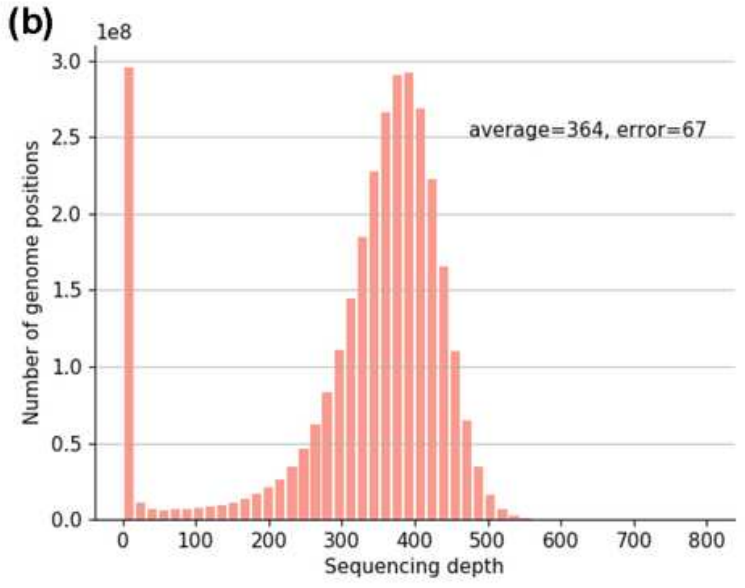

(c) Sequencing depth

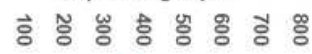

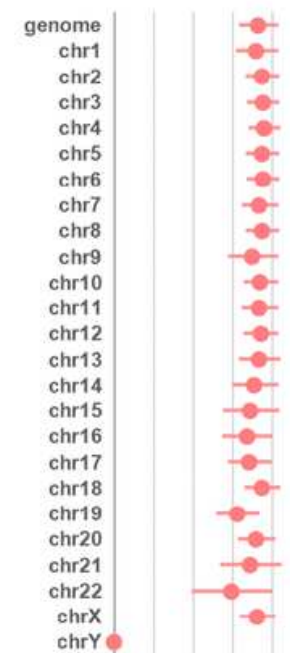

(d)

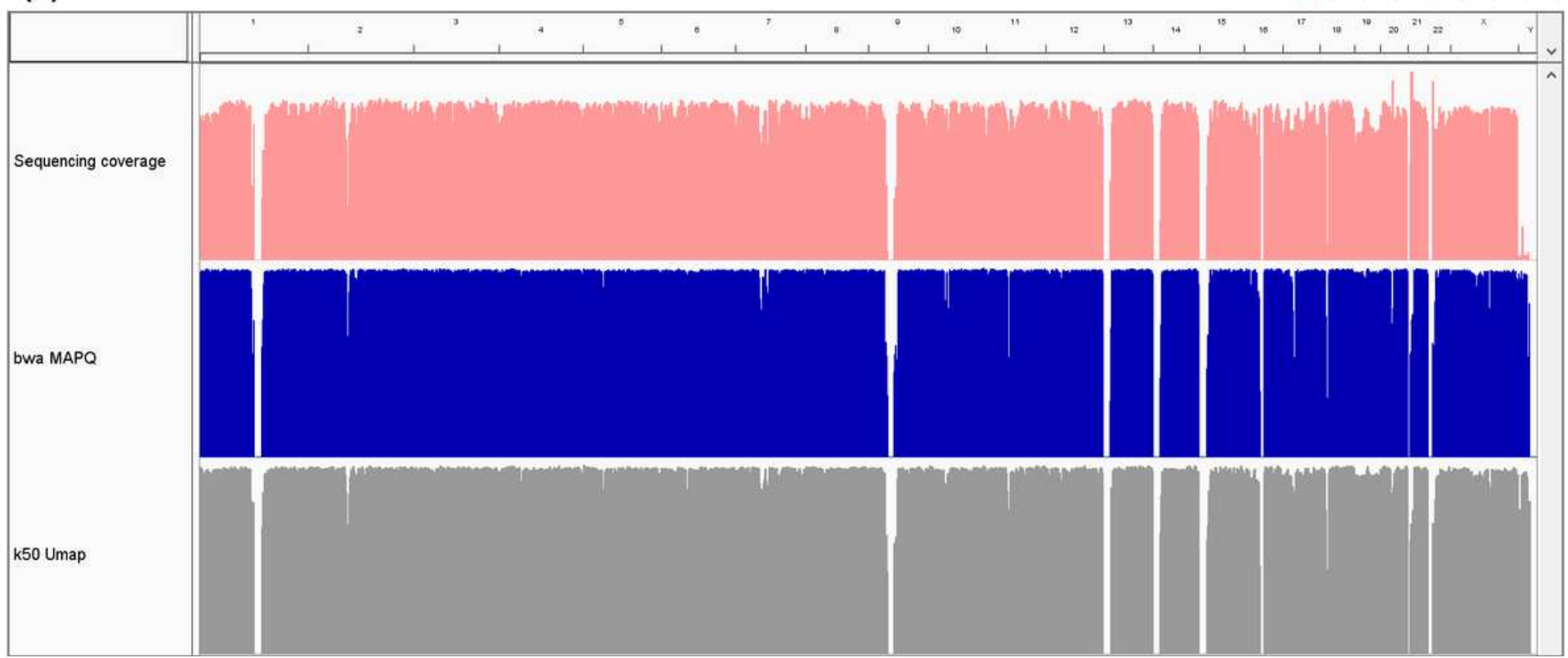

\section{Figure 1}

Distributions of genome coverage and sequencing depth of the NIPT dataset. (a) Average genome coverage and sequencing depth per sample and from all samples combined. (b) Summary histogram of sequencing depth over all genome positions. (c) Distribution of sequencing depth per chromosome. (d) IGV tracks of sequencing depth, bwa MAPQ score, and Umap k50 mappability across the whole genome. 
(a)

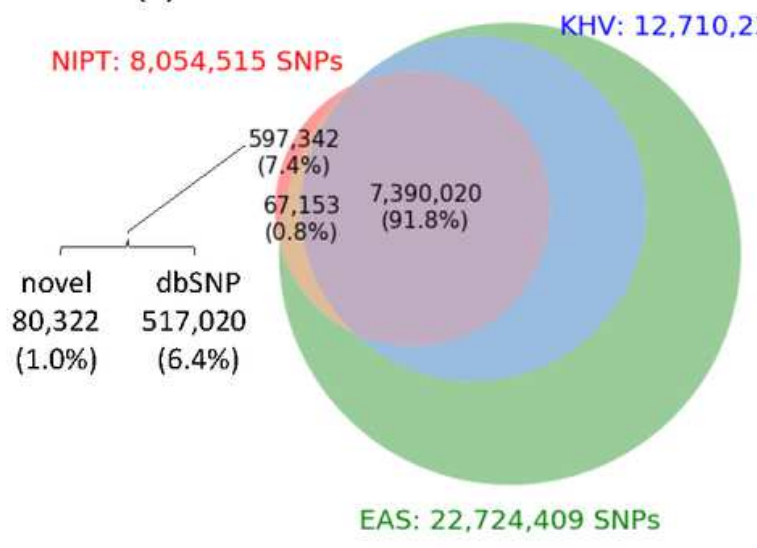

(c)

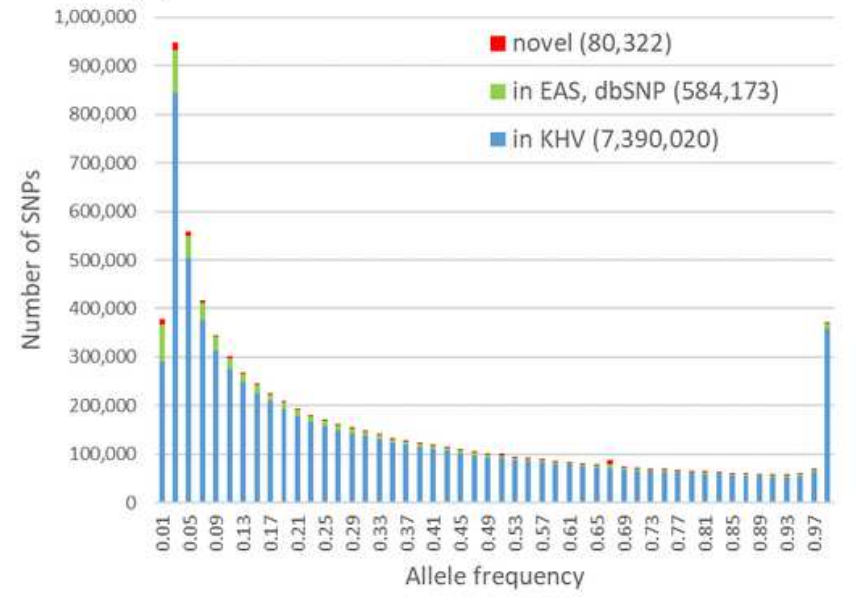

(b)

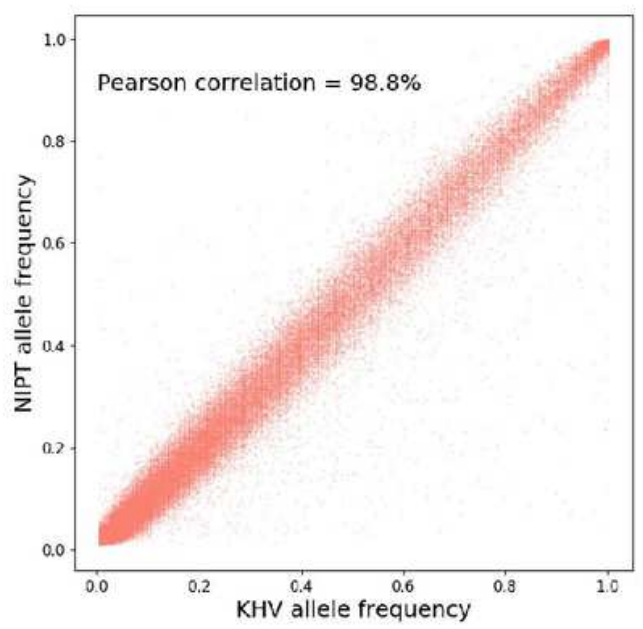

(d)

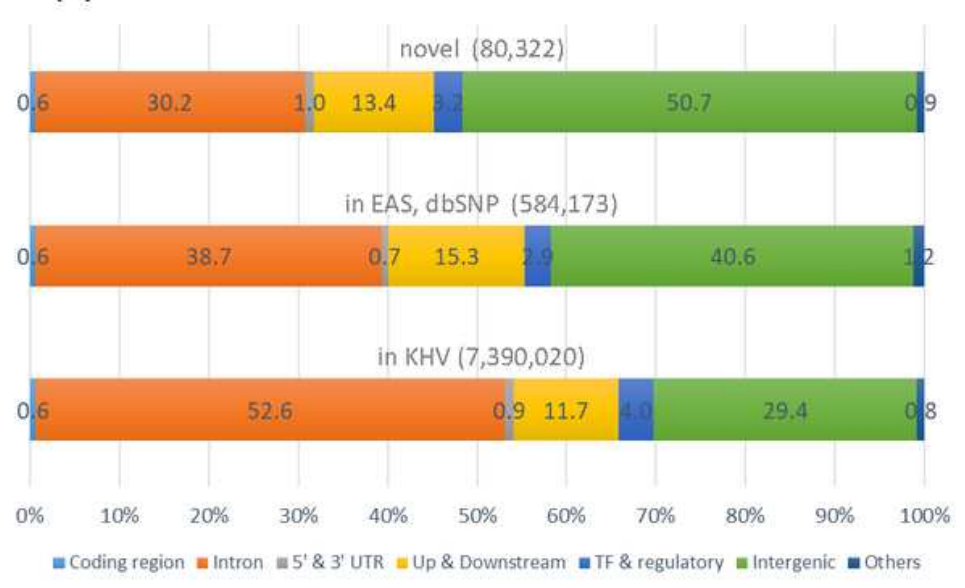

\section{Figure 2}

Summary of the NIPT call set. (a) Venn diagram comparison between the NIPT call set, the KHV and EAS call sets from the 1000 genomes project, and the dbSNP database. The percentages were calculated with respect to the NIPT call set. (b) Scatter plot comparison of allele frequency estimated from the NIPT and the KHV call sets. (c) Allele frequency distribution of the NIPT call set. (d) Distribution of locations and effects of variants in the NIPT call set.

\section{Supplementary Files}

This is a list of supplementary files associated with this preprint. Click to download.

- Manuscript.suppfigurestables.pdf

- Tables12.pdf 